\title{
Human infections caused by free-living amoebae
}

\author{
Katarzyna Król-Turmińska', Alina Olender ${ }^{1}$ \\ ${ }^{1}$ Chair and Department of Medical Microbiology Medical University of Lublin \\ Król-Turmińska K, Olender A. Human infections caused by free-living amoebae. Ann Agric Environ Med. 2017; 24(2): 254-260. doi: \\ $10.5604 / 12321966.1233568$
}

\begin{abstract}
Introduction. Among free-living amoebae that are widely distributed in nature only four genera/species are known as agents of human infections: Acanthamoeba spp., Naegleriafowleri, Balamuthia mandrillaris and Sappiniapedata. These amoebae are not well adapted to parasitism, and could exist in the human environment without the need for a host. Infections due to these amoebae, despite low morbidity, are characterized by relatively high mortality rate and pose serious clinical problems. Objectve. This review study presents and summarizes current knowledge about infections due to pathogenic and opportunistic free-living amoebae focused on epidemiology, clinical manifestations, diagnosis and treatment based on global literature.

State of knowledge. All four genera have been recognized as etiologic factors of fatal central nervous system infections and other serious diseases in humans. N. fowleri causes an acute fulminating meningoencephalitis in children and young adults. Acanthamoeba spp. and B.mandrillaris are opportunistic pathogens causing granulomatous amoebic encephalitis and disseminated or localized infections which could affect the skin, sinuses, lungs, adrenals and/or bones. Acanthamoeba spp. is also the main agent of acute eye infection - Acanthamoeba keratitis, mostly in contact lens wearers. However, there is only one recognized case of encephalitis caused by S. pedata.

Conclusions. Amoebic diseases are difficult to diagnose which leads to delayed treatment, and result in a high mortality rate. Considering those issues, there is an urgent need to draw more attention to this type of diseases.
\end{abstract}

Key words

Acanthamoeba, Naegleriafowleri, Balamuthia mandrillaris, amebiasis, protozoal infections

\section{INTRODUCTION}

Diseases caused by free-living protozoa pose serious clinical problems. New cases are constantly being reported worldwide. These kind of infections, despite low morbidity, are characterized by relatively high mortality rate, which is a huge challenge for efficient diagnosis and therapy $[1,2]$. One group of these pathogenic and opportunistic protozoa are free-living amoebae (FLA). FLA are aerobic, mitochondriate, eukaryotic microorganisms widely distributed in nature and the human environment. From many genera of free-living amoebae that exist in nature, only four are involved in human and animal infections. Among them there are several of Acanthamoeba species; however, only one species of each genera: Balamuthia (B.mandrillaris), Naegleria(N.fowleri) and Sapinia $(S$. pedata) causes infections $[1,3]$. These amoebae are ubiquitous, and have been found in water, soil and air, but also in sewage, swimming pools, flowerpots, water tubs, humidifiers, aquaria, eye wash solutions and hospital environment, e.g. dialysis and dental treatment units $[1,4]$. These protozoa are called amphizoic because of their ability to complete their life cycle inside host organism as well as in the environment. Human infections with these amoebae are rare but have been reported all over the world [3]. FLA can cause localized systemic diseases as well as disseminated infections. All four genera cause acute and almost always fatal infections of the central nervous system (CNS). Some of them are also responsible for extra-CNS infections of the skin, eyes, sinuses, lungs and kidneys. Diseases occur both in immunocompetent and immunocompromised individuals, including patients with AIDS [5]. Of greatest concern is the comparatively high

Address for correspondence: Katarzyna Król-Turmińska, Chair and Department of Medical Microbiology Medical University of Lublin

E-mail: katarzyna.k.krol@gmail.com

Received: 25 May 2016; accepted: 12 Jule 2016; first published on February 2017 mortality rate between infected individuals and lack of wellestablished recommended treatment. Early diagnosis of these infections is very important although it may be difficult due to non-specific symptoms.

Central nervous system infections. All four pathogenic FLA are known to cause infections of the central nervous system. Balamuthia mandrillaris (the only known species of Balamuthia), Naegleriafowleri, Sapiniapedata and several species of Acanthamoeba (e.gA.castellanii, A.culberstoni, A. polyphaga, A. hatchetti, A. rhysodes) induce CNS diseases in humans and animals. Naegleriafowleri causes fatal fulminating infection called primary amoebic meningoencephalitis (PAM), mainly in healthy children and young adults. Acanthamoebaand Balamuthia mandrillaris are responsible for granulomatous amoebic encephalitis (GAE), and chronic infection in both immunocompromised and immunocompetent hosts. To date, only a single case of encephalitis caused by S.pedata has been described in an immunocompetent male in Texas (Tab. 1) [1, 3].

Primary Amoebic Encephalitis (PAM). Naegleriafowleri is an thermophilic, free-living amoeboflagellate spread worldwide in most types of warm water reservoirs. Naegleria almost invariably causes fatal acute fulminating hemorrhagic infection - primary amebic meningoencephalitis (PAM). Since the first described case in the 1960s, PAM has been reported in more than 16 countries. The total number of cases is unknown. It is estimated that approximately 300 cases have occurred worldwide. The largest series of well-documented PAM cases comes from the USA, where 111 PAM cases have been reported [6]. In Europe, the first cases of PAM occurred in 1986in the territory of former Czechoslovakia. Since then, new cases have been reported also from Germany, France, Belgium, Hungary and Iran $[3,8]$. Infection due to 

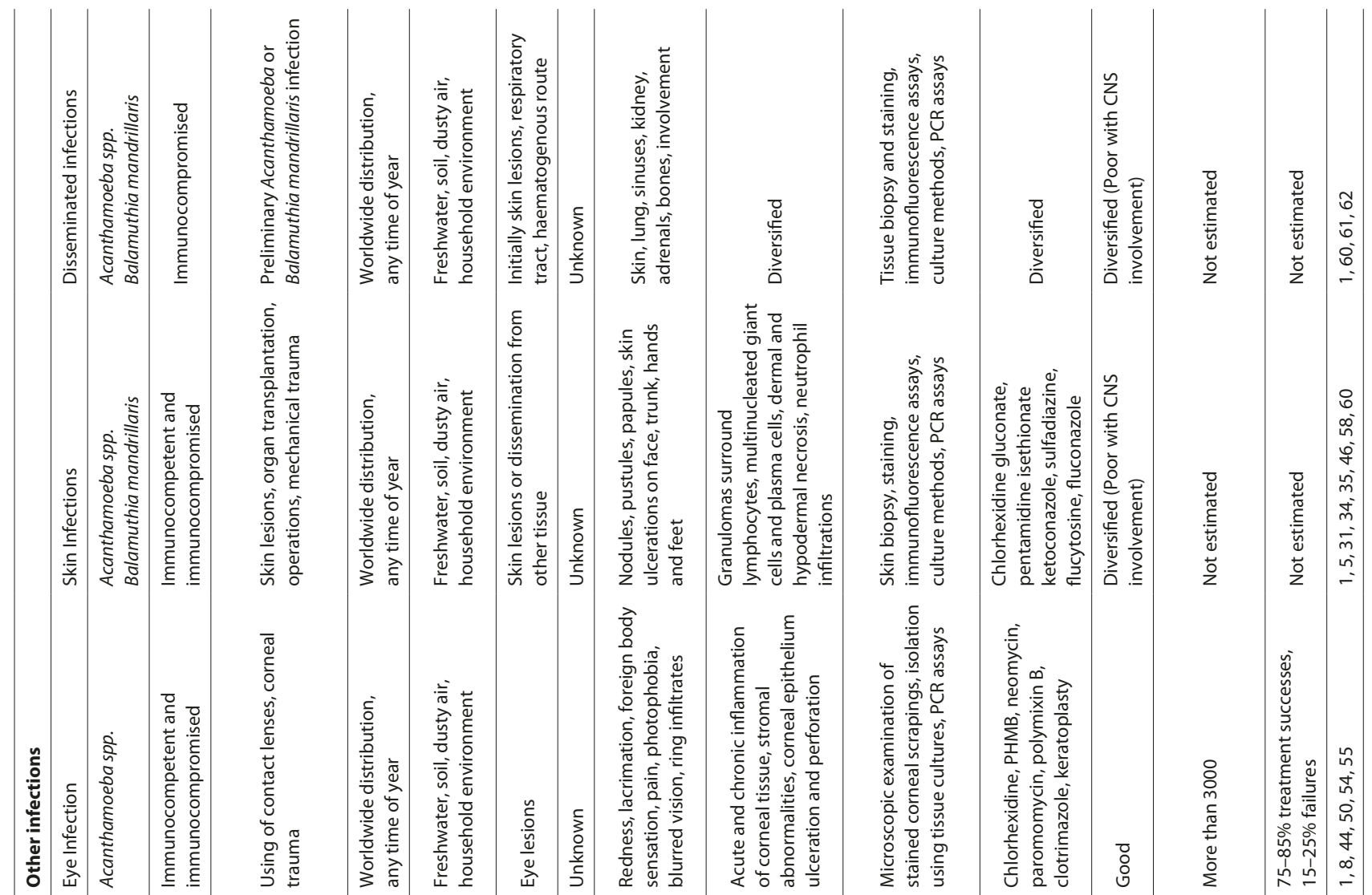

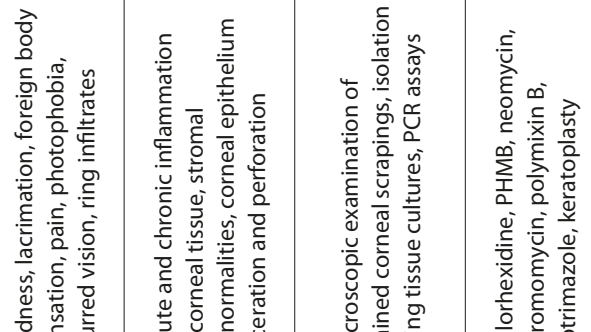

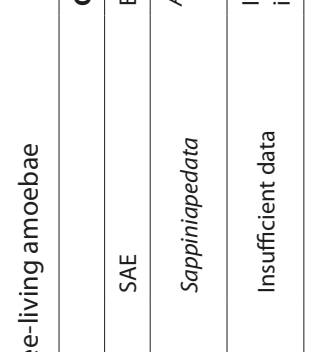

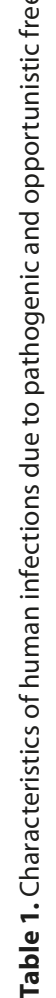

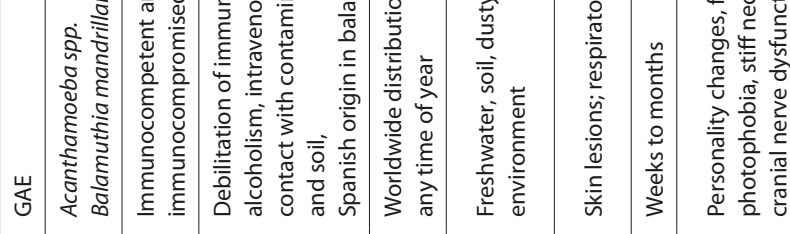

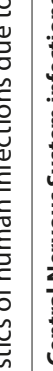
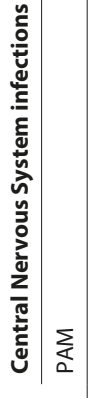

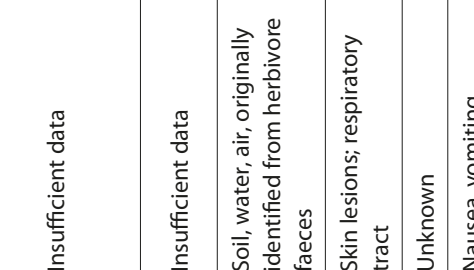

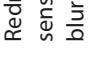

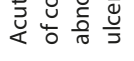

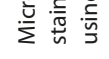

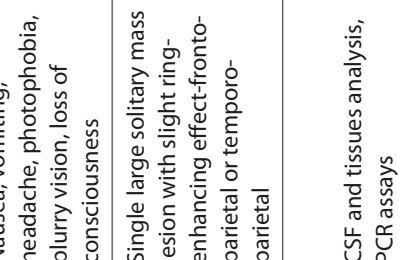

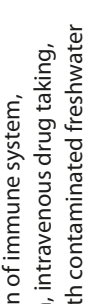
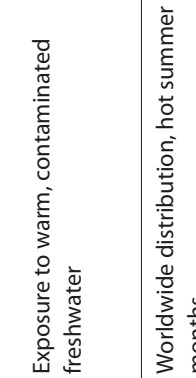

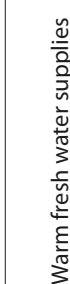
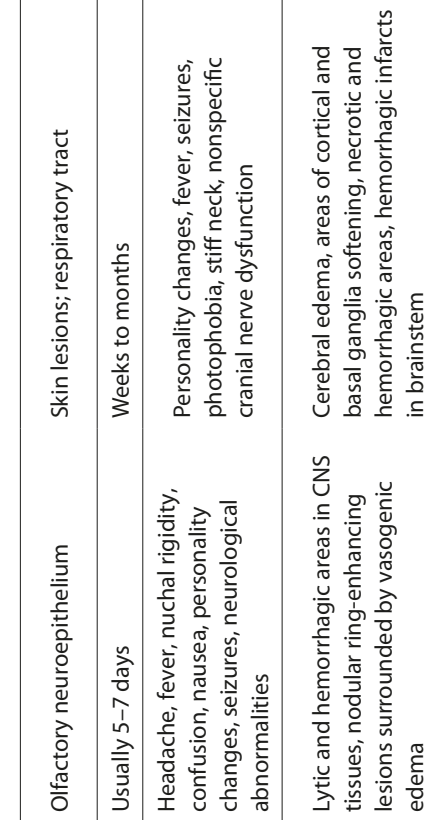

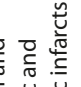

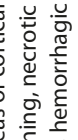

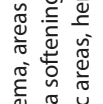

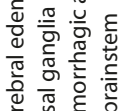

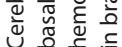

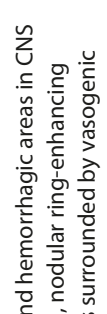

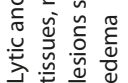

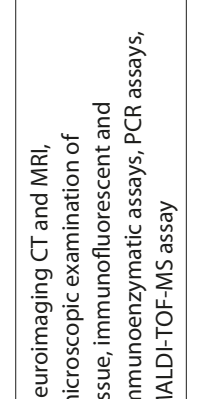

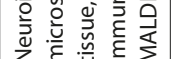

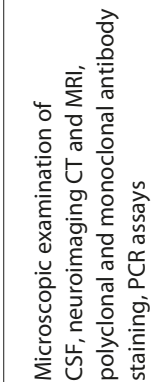

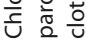

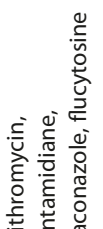

妾言产

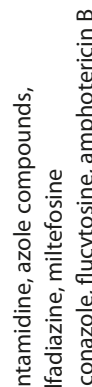

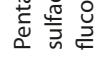

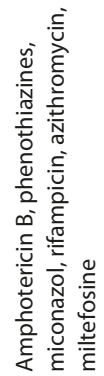

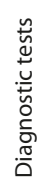

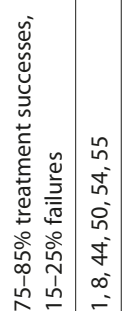

党

홓

잉

ฮั)

ปे ᄒे

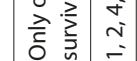

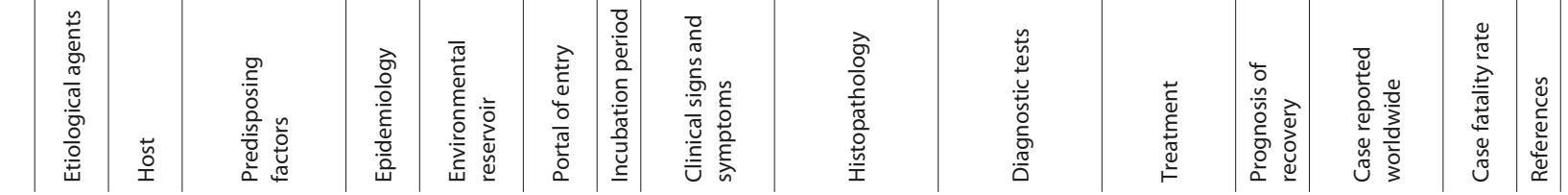


N.fowleri is acquired principally by healthy children and young adults with a history of recent exposure to warm contaminated freshwater, such as heated swimming pools, geothermal waters, warm ponds, or sewage systems carrying away industrial cooling water [1].

Pathophysiology and clinical manifestations. PAM occurs typically in the hot summer months during recreational activities - swimming or bathing in polluted water. Transmission to humans occurs primarily through inhalation of infested water. Increased contact of the nasal mucosa with infested water predispose humans to infection. Most casualties are young adults and children with a history of contacts with water containing the amoebae, about a week prior to the onset of neurological symptoms. The onset of illness occurs within 5-7 days and may develop rapidly after 24hrs. Because of the lack of distinctive clinical features, PAM can be easily confused with pyogenic, bacterial or viral meningitis. For that reason, any information about previous contact with warm water is essential $[1,9]$. The portal entry into the CNS is the olfactory neuroepithelium reached by the nasal passages. The amoebic trophozoites penetrate the olfactory mucosa and migrate along the olfactory nerve, crossing the cribriform plate and reach the olfactory bulbs. Trophozoites induce an inflammatory response associated with lytic necrotic haemorrhage. Then, numerous superficial haemorrhagic areas are seen in the cortex. Lesions are observed mainly around the base of the orbitofrontal and temporal lobe, base of the brain, hypothalamus, midbrain, pons, medulla oblongata, and the upper part of the spinal cord $[3,11]$. The earliest symptoms are bifrontal or bitemporal headaches, high temperature $\left(38.5-41^{\circ} \mathrm{C}\right)$, nuchal rigidity with positive Kernig and Brudzinski signs, followed by nausea, vomiting and behavioural abnormalities, such as irritability and restlessness. Subsequently, photophobia and neurological abnormalities develop, including lethargy, seizures, confusion, coma, diplopia or bizarre behaviour may occur. Cranial nerve palsies (third, fourth and six cranial nerves) may indicate cerebral oedema and herniation. Intracranial pressure is usually raised to $600 \mathrm{~mm} \mathrm{H}_{2} \mathrm{O}$, or higher. Cardiac rhythm abnormalities and myocardial necrosis may occur in some cases. Increased intracranial pressure and herniation usually lead to death $[1,9,10,11]$. In early stages of disease, the cerebrospinal fluid (CSF) may be grey to yellow-white, or red if it contains red cells. In the advanced stage, the red blood cell number increases to 24,600 cells $/ \mathrm{mm}^{3}$. The white blood cell count, predominantly polymorphonuclear leukocytes (PMN), may range from 300 cells $/ \mathrm{mm}^{3}$ to 26,000 cells $/ \mathrm{mm}^{3}$. In addition, protein concentration may vary from $100 \mathrm{mg} / 100 \mathrm{~mL}$ to $1,000 \mathrm{mg} / 100 \mathrm{~mL}$, and glucose may be $10 \mathrm{mg} / 100 \mathrm{~mL}$, or lower. The CSF pressure is usually very high, ranging from $300-600 \mathrm{mmHg}$. Prognoses are weak, death occurs generally within 7-10 days following infection $[9,11,12,13]$. Because of rapid onset and progression of PAM in humans, there is meager chance of effective humoral response to develop against the amoebae. Seidel et al. [14] described a case of 9-year-old who survived PAM. In this patient, anti-Naegleria antibodies of the IgM class were found [5]. Marciano-Cabral et al. [15] have demonstrated that large numbers of humans are exposed to both nonpathogenic and pathogenic Naegleria when they screened human sera for antibodies against amoeba. The sera collected from several individuals with a history of swimming in freshwater revealed not only IgM but also IgG antibodies. Whether these antibodies are protective is still unclear $[5,10]$.

Diagnosis. PAM should be suspected in children and young adults with acute neurological symptoms. A history that describes exposure to warm, freshwater is suggestive. The method of choice in early diagnosis is direct microscopic examination of the CSF as a centrifuged wet mount. The CSF may reveal the presence of motile amoebae which can be differentiated from host cells by staining fixed preparations with Wright's, Giemsa, trichrome or hematoxylin and eosin (H \& E). Gram stain is not useful for diagnosis because it does not show the characteristic nuclear morphology of the amoebae. Furthermore, the CSF may have a relatively high pressure, low glucose and high protein concentration. In the early course of the disease, polymorphonuclear leukocytes (PMNs) can be found in the CSF, but not bacteria. A phase contrast microscope is useful to optimize visualization of the amoebae $[4,5,9]$. Neuroimaging methods as Computer Tomography scans (CT) or Magnetic Resonance Imagining (MRI) show lesions, although these are non-specific. Scans can show obliteration of the cisternae around the midbrain and the subarachnoid space over the cerebral hemispheres $[1,9]$. Microscopic examination of the cerebral hemispheres, brain stem, cerebellum, and upper part of the spinal cord reveal fibrino-purulent leptomeningeal exudates containing predominantly PMNs and a few eosinophils, macrophages, and some lymphocytes. Amoebic trophozoites are seen within oedematous and necrotic tissue and deeply in the Virchow-Robin spaces, mostly around blood vessels, with no inflammatory response. Cysts are not observed. Naegleriacan be identified by polyclonal and monoclonal antibody staining [5]. Molecular techniques are useful both in CSF screening and identification of $N$. fowleri in amoebae cultures from human tissues. Real-time PCR assays have been developed to detect Naegleria in clinical samples. Nested-PCR and PCR can identify the presence of amoeba in microbiological cultures. A PCR assay using primers for the complete ribosomal internal transcribed spacer region (ITS) has been developed that allows for the discrimination of Naegleria species, and a species specific assay allows for the detection of $N$. fowleri $[16,17,18,19]$.

Antimicrobial therapy. There are only a few examples of patients who have survived PAM. The drug of choice in treating PAM is the antifungal polyene antibiotic amphotericin. Naegleria are highly sensitive to this drug, but treatment must be started early in order to be effective. In a well-documented recovery, the patient was treated with intravenous and intrathecal amphotericin $\mathrm{B}$, miconazol and oral rifampicin. In vitro testing of the isolate from the patient showed no efficacy for rifampicin [14]. Amphotericin B was reported to be more effective against Naegleria than amphotericin methyl ester, a water-soluble form of the drug. All reported recoveries revealed that the patients were successfully cured by mixed treatment, but always with amphotericin B. Phenothiazine compounds (chlorpromazine and rifluoperazine), which can accumulate in the CNS, were tested in vitro and were found to have inhibitory effects on N. fowleri [20]. Azithromycin has been shown to be effective against Naegleria in a mouse model of disease both in vitro and in vivo [21]. Other macrolides are less effective. Naegleriafowleriis also sensitive to the triazole and voriconazole $[22,23]$. Moreover, miltefosine (breast 
cancer and anti-leishmania drug) was shown to be effective against free-living amoebae in laboratory tests [22].

Granulomatous amebic encephalitis (GAE). Granulomatous amebic encephalitis is a rare opportunistic infection caused by Balamuthia mandrillaris and several species of Acanthamoeba. Both free-living amoebae are distributed worldwide and can be found in freshwater, soil, dust and air, although Acanthamoeba is more ubiquitous. Acanthamoeba has been isolated from a wide variety of habitats, including, e.g. seawater, sewage, tap water, beach sands, home aquaria, humidifiers and hospital environment $[3,24]$. To date, more than 150 cases of GAE caused by Acanthamoeba have been reported in the literature, among them, 11 cases of immunocompetent hosts [25]. Approximately 200 cases of Balamuthia GAE have been reported from all continents except from Africa. Balamuthiasis appears to occur more frequently among patients of Spanish origin, probably because of genetic susceptibility or environmental exposure [27,23]. A fatal case of multiple protozoan infection (Acanthamoebaspp, Balamuthia mandrillaris, and Toxoplasma gondii) in an HIVinfected patient has also been reported [29].

Pathophysiology and clinical manifestations. GAE is an opportunistic and fatal disease which can occur any time of the year. The portal of entry for both organisms are either through skin lesions contaminated with soil, or as a cyst taken into the respiratory tract. An association between water contact and infection has not been reported, but it could be a possible route of infection. Acanthamoeba GAE occurs, most often, in humans with compromised metabolic, physiologic, or immunologic functions because of HIV/AIDS or in those who are chronically ill, diabetic, have undergone organ transplantation, or are otherwise debilitated. Balamuthia GAE can occur both in healthy individuals and immunocompromised patients. The risk factors are alcoholism, intravenous drug taking, contact with contaminated freshwater and soil $[2,5,31]$. After primary exposure, both amoebae invade the blood stream and spread haematogenously to the brain. They can also reach other organs, e.g. the lungs, sinuses or the skin [32]. In the case of balamuthiasis, two patterns of clinical presentation have been described: some patients develop an initial skin lesion followed by development of neurologic manifestations within weeks or months, while others present first with the CNS involvement $[33,34]$. Incubation period is unclear as it is often difficult to determine when exposure took place. The onset of disease is slow and subtle and symptoms develop within several weeks to months. In balamuthiasis it can be shorter and range from 1-30 days [2, 3, 31]. The typical features of GAE include behavioural and personality changes, hemipharesis, aphasia, fever, seizures, photophobia, vision loss, stiff neck, non-specific cranial nerve dysfunction, including headache, nausea, vomiting, increased intracranial pressure or loss of consciousness. In acanthamebiasiscoma, facial asymmetry is also often observed. The clinical signs of GAE are not specific. The disease is often misdiagnosed as bacterial or viral encephalitis or brain tumour $[1,11,20$, 35]. The pathophysiology of Acanthamoeba and Balamuthia CNS infections is similar. Amoebae are usually not found in the CSF, although in a few cases they have been isolated from the CSF $[20,36]$. Autopsies of the brain reveal cerebral oedema, areas of cortical and basal ganglia softening, and multiple necrotic and haemorrhagic areas in the CNS tissues. The brainstem, cerebral hemispheres and the cerebellum may show areas of haemorrhagic infarcts. The haemorrhagic necrosis and inflammatory infiltrates consist of neutrophils, lipid-containing macrophages, mononuclear cells, and multinucleated giant cells. Multinucleated giant cells forming granulomas are seen in tissue from immunocompetent patients, but less often in immunocompromised individuals. Some patients, mostly with balamuthiasis, develop chronic ulcerative skin lesions or erythematous nodules. Both amoebic trophozoites and cysts are found within all infected tissues $[1,37]$. In both infections, the CSF examination reveals lymphocytic pleocytosis, increased protein concentration and normal to low glucose concentration $[35,38]$.

Diagnosis. Brain lesions can be detected by neuroimaging methods such as CT and MRI, but the results are not specific and these methods are of limited diagnostic value for GAE. Microscopy and haematoxylin-eosin staining of hosts brain tissue can be used to detect trofozoites and cysts, but morphological features are inadequate for differentiating Acanthamoeba from $B$. mandrillaris. The differentiation of these amoebae from macrophages is quite problematic and requires expertise. Humoral response to Acanthamoeba and Balamuthia is well developed. Anti-Acanthamoebaand anti-B.mandrillaris antibodies have been found in the sera of healthy and GAE infected individuals. Consequently, several immunodiagnostic tests have been well developed and produced good results. Immunofluorescent microscopy, indirect immunofluorescent and immunoenzymatic assays can be successfully used to examine serum and tissue samples. Acanthamoeba can be isolated from patients' tissue and cultured in vitro, but this method is not recommended for $B$. mandrillaris $[2,4]$. Molecular techniques, such as PCR and real-time PCR, have also been used to identify Acanthamoeba and Balamuthia in the CSF, brain and other tissues. A real-time multiplex PCR assay has been developed for simultaneous detection of Acanthamoeba spp., Balamuthia mandrillaris and Naegleriafowleriin clinical specimens within five hours [18]. In addition, matrix-assisted laser desorption-ionization time of flight MS (MALDI-TOFMS) may be of potential value in quick identification of $B$. mandrillaris in clinical specimens [1].

Antimicrobial therapy. The treatment of GAE is problematic because of non-specific symptoms and lack of a good reliable diagnostic test. GAE is often detected post-mortem. Moreover, optimal management modality remains uncertain. A number of drugs have demonstrated in vitro activity against Acanthamoeba and have resulted in success in a few patients when used alone or in combination: rifampicin, azoles (fluconazole, itraconazole, voriconazole), pentamidine isethionate, sulfadiazine, flucytosine, azithromycin, miltefosine, and caspofungin [5, 35, 37]. A few patients with Balamuthia GAE were successfully treated with a combination of pentamidine, 5-flucytosine, fluconazole and a macrolide (clarithromycin or azithromycin) with one of the following: sulfadiazine, miltefosine, thioridazine or liposomal amphotericin B. In Peru, five patients have also been successfully treated with a combination of fluconazole or itraconazole in addition to albendazole and miltefosine $[31,37$, $41,42]$. Currently, there is scarce data regarding treatment and no controlled studies to guide optimal antimicrobial therapy. 
Sappinia Amoebic Encephalitis (SAE). The first and only case of amoebic encephalitis caused by Sappiniapedata (originally identified as S.diploidea) was described in 2001 by Gelmanet al. [43]. The patient was a hitherto healthy immunocompetent 38-year-old male farmer from Texas who had contact with farm animals and faecal-contaminated soil. The infection may have developed from an earlier sinus infection. The patient developed nausea, vomiting, bifrontal headache, photophobia, blurry vision, and loss of consciousness. MRI detected a $2 \mathrm{~cm}$ mass in the left temporal lobe with slight ring enhancement. The lesion was excised and section of the brain tissue showed trophozoites with distinctive double nuclei. Basing on that, it was possible to differentiate Sappinia from other free-living amoebae $[2,4]$. Sappinia spp. can be identified by molecular assays, e.g. PCR (e.g. ITS PCR, real-time PCR). The assay specific for Sappinia can be incorporated into already existing multiplex PCR assay described by Qvarnstromet al. $[2,18]$. The patient was treated with azithromycin, pentamidiane, itraconazole, and flucytosine [43]. Sappinia spp. has been reported in Europe, North America, Egypt, Japan, the Middle East and West India [1].

Eye infections - Acanthamoeba keratitis (AK). Acanthamoeba keratitis is an acute localized infection involving the cornea. Unlike GAE, the disease occurs worldwide in immunocompetet individuals following corneal trauma or more commonly in contact lens users (Tab. 1) $[36,44]$. Among the species that have been implicated as etiological agents are A. castellanii, A. polyphaga, A. rhysodes, A. culbertsoniand $A$. hatchetti[5]. The main risk factors associated with AK include using contaminated contact lens solutions, wearing contact lens for extended periods of time, using all-in-one lens solutions, showering while wearing contact lenses, and poor contact lens hygiene [45]. The number of affected individuals is increasing worldwide which correlates with rising number of contact lens users. Previous studies found the prevalence of 1.2 cases per million adults and 0.2 (USA) to 2 (UK per 10,000 soft contact lens wearers per year [46]. A dramatic rise in the incidence of AK was seen in the 1980s, which was largely connected with increased adoption of wearing soft contact lens, and the use of non- sterile contact lens solutions and homemade saline tablets. Additional outbreaks in the late 1990s and 2000s were reported in the USA and in Europe [47]. Moreover, recent outbreaks of AK have been observed in the USA and Australia $[48,50]$.

Clinical manifestations. Clinical manifestation starts with redness, lacrimation, epiphora, diptosis, conjunctival hyperhaemia, foreign body sensation, pain, photophobia and appearance of ring infiltrates. Epithelial irregularities and pseudodendritic epithelial lesions represent anearly stage of the disease. Typically, one eye is involved; however, bilateral keratitis has also been described [52]. A variety of proteases produced by Acanthamoebatrofozoites causes parasitemediated cytolysis of the cornea. The infection destroys the corneal epithelium and stroma, causing infiltration by inflammatory cells. Stromal abnormalities like nummular infiltrates and radial keratoneuritis can occur. Keratoneuritis is characterized by linear, radial, branching infiltrates of the parasite along the corneal nerves into the anterior stroma, with anterior chamber reaction leading to hypopyon. A ringshaped stromal infiltrate is a characteristic sign of advanced infection. If not diagnosed early and treated promptly, many complications can develop in the later stage of the disease, like corneal epithelial ulceration, perforation, loss of visual acuity and eventually blindness and enucleation $[53,54$, $55,56]$. AK may be confused with viral keratitis (e.g. due to HSV) resulting in delayed correct diagnosis and late initiated therapy [5].

Diagnosis. Diagnosis is based on identification of trophozoites and cyst in the corneal scrapings and biopsy specimens. Trophozoites can be visualized by staining of cornea scrapings with calcofluor. Scrapings can be also cultured and examined microscopically for the presence of amoeba. Confocal microscopy can also aid diagnosis. Molecular methods (PCR, real-time PCR) have also been used, and generally they are more sensitive than specific stains and cultures $[5,11]$.

Treatment. Treatment of AK is more successful that than of GAE. Several varieties of drugs can be used, including chlorhexidine, polyhexamethylenebiguanide, propamidine, isethionate, dibromopropamidine isethionate, neomycin, paromomycin, polymixin B, clotrimazole, and itraconazole. Significant success has been achieved with either polyhexamethylenebiguanide (PHMB) or chlorhexidine gluconate with or without propamidineisethiocyanate. When medical treatment failed, debridement and/or penetrating keratoplasty have been used, with good results in some cases. Application of steroids is common, both to relieve pain and to lessen inflammation, particularly following keratoplasty $[1,11,44]$.

Other infections. In addition to GAE, Acanthamoeba and Balamuthia mandrillarisare etiological factors of localized and disseminated infections including skin, lung, sinus, kidneys or adrenal abscesses (Tab. 1) [1, 5]. These infection are rare and usually connected with organ transplantation and other conditions that may affect the host immune system. Cutaneous acanthamebiasis is an uncommon opportunistic infection, particularly in immunocompromised patients with HIV, with or without CNS involvement. Cutaneous disease has also been reported in non-HIV-infected patients and immunocompetent hosts $[35,47,57]$. Risk factors for Acanthamoeba skin infection include traumatized areas, such as surgical scars, viral lesions, bites and mechanical trauma. Characteristic lesions are nodules, pustules, papules and skin ulcerations that contain Acanthamoebatrophozoites and cysts. The most frequently lesion affected sites are the face, trunk and extremities [58]. Diagnosis of Acanthamoeba skin infection includes tissue histology, staining the smears of tissue, immunofuorescence assays, culture on non-nutrient agar plates seeded with bacteria as food source or tissue culture. More recently, PCR assays have been used. Treatment includes application of chlorhexidine gluconate and ketoconazole cream with one of the following: pentamidine isethionate, sulfadiazine, flucytosine, fluconazole or itraconazole $[11,58]$. The majority of patients with GAE due to Balamuthia exhibit skin ulcerations that may be several centimeters wide and appear on the face, trunk, hands and feet of patients [31]. Moreover, several reports have described Balamuthia GAE preceded by initial cutaneous infections. In many cases, there was a history of previous trauma. Treatment of cutaneous balamuthiasis is similar to GAE 
treatment. The skin ulceration may provide an opportunity for the early diagnosis of the CNS infection. Histopathologic characteristics include the presence of granulomatous reaction. Granulomas are surrounded by an infiltrate rich in lymphocytes and plasma cells. Multinucleated giant cells may be present inside and outside granulomas $[34,59]$. Diagnosis includes skin biopsy, staining, indirect immunofluorescence assays and culture methods. A PCR technique for the detection of amoebic DNA material in human tissue has also been developed $[34,60]$. In amoebic infection, other organs outside the CNS might be involved by the haematogenous route. Disseminated infections could affect several areas, such as the skin, lung, sinuses, kidney, or even bones, causing osteomyelitis [61]. Acanthamoeba infections of the nasal mucosa and paranasal sinuses are very rare and have been described in severely immunosuppressed patients with HIV. Pulmonary involvement can be bilateral with patchy infiltrates and usually occurs with other tissue infection. Diagnosis generally has been made post-mortem by observing trophozoites and cysts in the lung tissue samples [63].

\section{CONCLUSIONS}

The presented study describes human infections due to free-living amoebae: Acanthamoebaspp., Naegleriafowleri, Balamuthia mandrillaris and Sapiniapedata. The main aim of this study was to review and summarize current knowledge about these kinds of infections based on global literature. The epidemiology, clinical manifestations, diagnosis and treatment have been described. Another goal was to draw attention to the growing problem of FLA diseases in healthy and immunocompromised people. The number of infections caused by FLA is relatively low, possibly due to difficulties in diagnosis. It is likely that many cases have gone unrecognized. However, difficult diagnosis, lack of awareness, limited efficiency of treatment, resistance of antimicrobials, and finally the abundance of more prominent diseases resulting in often fatal consequences make these infections a great public health concern. The number of confirmed and reported amoebic diseases rises each year. In many cases, early diagnosis is crucial to successful treatment. Considering these issues, there is an urgent need to improve diagnostic and treatment trials.

\section{REFERENCES}

1. Visvesvara GS, Moura H, Schuster FL. Pathogenic and opportunistic free-living amoebae: Acanthamoeba spp., Balamuthia mandrillaris, Naegleriafowleri and Sappiniadiploidea. FEMS Immunol Med Mic. 2007; 50(1): 1-26.

2. Diaz JH. Increasing intracerebral infections caused by free-living amebae in the United States and Worldwide. J Neuroparasitol. 2010; 1(1): 1-10.

3. Schuster FL, Visvesvara GS. Free-living amoebae as opportunistic and non-opportunistic pathogens of humans and animals. Int J Parasitol. 2004; 34: 1001-1027.

4. da Rocha-Azevedo B, Tanowitz HB, Marciano-Cabral F. Diagnosis of Infections Caused by Pathogenic Free-Living Amoebae. InterdiscipPerspect Infect Dis 2009; 2009: 1-14.

5. Visvesvara GS. Free-living amebae as opportunistic agents of human disease. J Neuroparasitol. 2010; 1: doi: 10.403/jnp/N100802

6. Yoder JS, Eddy BA, Visvesvara GS, Capewell L, Beach MJ. The epidemiology of primary amoebic meningoencephalitis in the USA, 1962-2008. Epidemiol Infect. 2010; 138(7): 968-975.
7. Yoder SJ, Straif-Bourgeois S, Roy SL, Moore TA, Visvesvara GS, Ratard $\mathrm{RC}$, et al. Primary Amebic Meningoencephalitis deaths associated with sinus irrigation using contaminated tap water. Clin Infect Dis. 2012; 55: 79-85.

8. Niyyati M, Lasjerdi Z, Zarein-Dolab S, Nazar M, Behniafar H, Mahmoudi MR, NazemalhosseiniMojarad E. Morphological and Molecular Survey of Naegleria spp. in Water Bodies Used for Recreational Purposes in Rasht city, Northern Iran. Iran J Parasitol. 2015;10(4):523-529.

9. Martinez AJ. Free-living amebas: natural history, prevention, diagnosis, pathology, and treatment of disease. CRC Press Inc. Boca Raton, Florida 1985.

10. Capewell LG, Harris AM, Yoder JS, Cope JR, Eddy BA, Roy SL, Visvesvara GS, Fox LM, Beach MJ. Diagnosis, Clinical Course, and Treatment of Primary Amoebic Meningoencephalitis in the United States, 1937-2013. J Pediatric Infect Dis Soc. 2015; 4(4): 68-75.

11. Trabelsi H, Dendana F, Sellami A, Sellami H, Cheikhrouhou F, Neji $S$, et al. Phatogenic free-living amoebae: Epidemiology and clinical review. PathologieBiologie. 2012; 60: 399-405.

12. Visvesvara GS, Maguire JH. Pathogenic and opportunistic freeliving amebas. Acanthamoeba spp., Balamuthia mandrillaris, Naegleriafowleri, and Sappiniadiploidea. Tropical Infectious Diseases, Vol. 2 (GuerrantRL, WalkerDH\&WellerPF, eds) Churchill Livingstone. 2006: 1114-1125.

13. Kemble SK, Lynfield R, DeVries AS, Drehner DM, Pomputius WF, Beach MJ, et al. Fatal Naegleriafowleri infection acquired in Minnesota: possible expanded range of a deadly thermophilic organism. Clin Infect Dis. 2012; 54(6): 805.

14. Seidel JS, Harmatz P, Visvesvara GS, Cohen A, Edwards J, Turner J. Successful treatment of primary amebic meningoencephalitis. N Engl J Med. 1982; 306: 346-348.

15. Marciano-Cabral F, Cline ML, Bradley SG. Specificity of antibodies from human sera for Naegleriafowleri. J ClinMicrobiol. 1987; 25: 692-697.

16. Pelandakis M, Pernin P. Use ofmultiplex PCR and PCR restriction enzyme analysis for detection and exploration of the variability in the free-living amoeba Naegleria in the environment. Appl Environ Microbiol. 2002; 68(4): 2061-2065.

17. Régoudis E, Pélandakis M. Detection of the freeliving amoeba Naegleria fowleri by using conventional and real-time PCR based on a single copy DNA sequence. ExpParasitol. 2016; 161: 35-39.

18. Qvarnstrom Y, Visvesvara GS, Sriram R, da Silva AJ. Multiplex realtime PCR assay for simultaneous detection of Acanthamoeba spp., Balamuthia mandrillaris, and Naegleriafowleri. J ClinMicrobiol. 2006; 44(10): 3589-3595.

19. Schild M, Gianinazzi C, Gottstein B, Muller N. PCRbased diagnosis of Naegleria sp. infection in formalin-fixed and paraffin-embedded brain sections. J ClinMicrobiol. 2007; 45(2): 564-567.

20. Schuster FL, Visvesvara GS. Opportunistic amoebae: challenges in prophylaxis and treatment. Drug Resist Update. 2004; 7: 41-51.

21. Goswick SM, Brenner GM. Activities of therapeutic agents against Naegleriafowleri in vitro and in a mouse model of primary amebic meningoencephalitis. J Parasitol. 2003; 89: 837-842.

22. Schuster FL, Guglielmo BJ, Visvesvara GS. In-vitroactivity of miltefosine and voriconazole on clinical isolates of free-living amebas Balamuthia mandrillaris, Acanthamoeba spp., and Naegleriafowleri. J EukaryotMicrobiol. 2006; 53: 121-126.

23. Schuster FL, Honarmand S, Visvesvara GS, Glaser C. Detection of antibodies against free-living amoebae Balamuthia mandrillaris and Acanthamoeba species in a population of patients with encephalitis. Clin Infect Dis. 2006; 42: 1260-1265.

24. Ahmad AF, Andrew PW, Kilvington S. Development of a nested PCR for environmental detection of the pathogenic free-living amoeba Balamuthia mandrillaris. J EukaryotMicrobiol. 2011; 58: 269-271.

25. Reddy R, Vijayasaradhi M, Uppin MS, Challa S, Jabeen A, Borghain R. Acanthamoeba meningoencephalitis in an immunocompetent patient: an autopsy case report. Neuropathol. 2011; 31(2): 183-187.

26. Latifi AR, Niyyati M, Lorenzo-Morales J, Haghighi A, SeyyedTabaei SJ, Lasjerdi Z.Presence of Balamuthia mandrillaris in hot springs from Mazandaran province, northern Iran. Epidemiol Infect. 2016; 18:1-6.

27. Diaz JH. The public health threat from Balamuthia mandrillaris in the southern United States. J La State Med Soc. 2011; 163(4): 197-204.

28. Gupte AA, Hocevar SN, Lea AS, et al. Transmission of Balamuthia mandrillaris through solid organ transplantation: utility of organ recipient serology to guide clinical management. Am J Transplant. 2014;14:1417-1424. 
29. Pietrucha-Dilanchian P, Chan JC, Castellano-Sanchez A, Hirzel A, Laowansiri P, Tuda C, et al. Balamuthia mandrillaris and Acanthamoeba amebic encephalitis with neurotoxoplasmosis coinfection in a patient with advanced HIV infection. J ClinMicrobiol. 2012; 50(3): 1128-1131.

30. Satlin MJ, Graham JK, Visvesvara GS, et al. Fulminant and fatal encephalitis caused by Acanthamoeba in a kidney transplant recipient: case report and literature review. Transpl Infect Dis. 2013;15:619-626.

31. Siddiqui R, Khan NA. Balamuthia amoebic encephalitis: An emerging disease with fatal consequences. Microb Pathogenesis. 2008; 44: 89-97.

32. Jung S, Schelper RL, Visvesvara GS, Chang HT. Balamuthia mandrillaris meningoencephalitis in an immunocompetent patient: an unusual clinical course and a favorable outcome. Arch Path Lab Med. 2004; 128: 466-468.

33. Reed RP, Cooke-Yarborough CM, Jaquiery AL, Grimwood K, Kemp AS, $\mathrm{Su}$ JC, Forsyth JR. Fatal granulomatous amoebic encephalitis caused by Balamuthia mandrillaris. Med J Aust. 1997; 167(2): 82-84.

34. Pritzker AS, Kim BK, Agrawal D, Southern PM Jr, Pandya AG. Fatal granulomatous amebic encephalitis caused by Balamuthia mandrillaris presenting as a skin lesion. J Am AcadDermatol. 2004; 50(2): 38-41.

35. Marciano-Cabral F., Cabral G. Acanthamoeba spp. as agents of disease in humans. ClinMicrobiol Rev. 2003; 16: 273-307.

36. Singhal T, Bajpai A, Kalra V, Kabra SK, Samantaray JC, Satpathy G, Gupta AK. Successful treatment of Acanthamoeba meningitis with combination oral antimicrobials. Pediatr Infect Dis J. 2001; 20(6): 623-627.

37. Martinez DY, Seas C, Bravo F, Legua P, Ramos C, Cabello AM, Gotuzzo E. Successful treatment of Balamuthia mandrillaris amoebic infection with extensive neurological and cutaneous involvement. Clin Infect Dis. 2010; 51(2): 7-11.

38. Schuster FL, Yagi S, Gavali S, Michelson D, Raghavan R, Blomquist I, et al. Under the radar: balamuthia amebic encephalitis. Clin Infect Dis. 2009; 48(7): 879.

39. Hajialilo E, Niyyati M, Solaymani M, Rezaeian M. Pathogenic FreeLiving Amoebae Isolated From Contact Lenses of Keratitis Patients. Iran J Parasitol. 2015; 10(4): 541-546.

40. Jackson BR, Kucerova Z, Roy SL, et al. Serologic survey for exposure following fatal Balamuthia mandrillaris infection. Parasitol Res. 2014;113:1305-1311.

41. Doyle JS, Campbell E, Fuller A, Spelman DW, Cameron R, Malham G, et al. Balamuthia mandrillaris brain abscess successfully treated with complete surgical excision and prolonged combination antimicrobial therapy. J Neurosurg. 2011; 114(2): 458-462.

42. Lobo SA, Patil K, Jain S, Marks S, Visvesvara GS, Tenner M, et al. Diagnostic challenges in Balamuthia mandrillaris infections. Parasitolo Res. 2013; 112(12): 4015-4019.

43. Gelman BB, Rauf SJ, Nader R et al. Amoebic encephalitis due to Sappiniadiploidea. J Am Med Assoc. 2001; 285: 2450-2451.

44. Seal DV. Acanthamoeba keratitis update-incidence, molecular epidemiology and new drugs for treatment. Eye. 2003; 17: 893-905.

45. Fraser MN, Wong Q, Shah L, Holland SP, Morshed M, Isaac-Renton $J$, et al. Characteristics of an Acanthamoeba keratitis outbreak in British Columbia between 2003 and 2007. Ophthalmol. 2012; 119(6): 1120-1125.

46. Parmar DN, Awwad ST, Petroll WM, Bowman RW, McCulley JP, Cavanagh HD. Tandem scanningconfocal corneal microscopy in the diagnosis of suspected Acanthamoeba Keratitis. Ophthalmol. 2006; 113(4): 538-547.

47. Page MA, William DM. Acanthamoeba Keratitis: A 12-Year Experience Covering a Wide Spectrum of Presentations, Diagnoses, and Outcomes. J Ophthalmol. 2013; 2013: 1-6.

48. Tu E, Joslin CE. Recent Outbreaks of Atypical Contact Lens-Related Keratitis: What Have We Learned? Am J Ophthalmol. 2010; 150: 602-608.

49. Morrison AO, Morris R, Shannon A, Lauer SR, Guarner J, Kraft CS. Disseminated Acanthamoeba Infection Presenting With Cutaneous Lesions in an Immunocompromised Patient: A Case Report, Review of Histomorphologic Findings, and Potential Diagnostic Pitfalls. Am J ClinPathol. 2016; 145(2): 266-270.

50. Patel DV, Rayner SC. Resurgence of Acanthamoeba keratitis in Auckland, New Zealand: a 7-year review of presentation and outcomes. Clin. Exp. Ophthalmol. 2010; 38: 15-20.

51. Verani JR, Lorick SA, Yoder JS, et al. National outbreak of Acanthamoeba keratitis associated with use of a contact lens solution, United States. Emerg Infect Dis. 2009;15:1236-1242.

52. Wilhelmus KR, Jones DB, Matoba AY, Hamill MB, Pfugfelder SC, Weikert MP. Bilateral Acanthamoeba Keratitis. Am J Ophthalmol. 2008; 145: 193-197.

53. Mannis MJ, Tamaru R, Roth AM, Burns M, Thirkill C. Acanthamoebasclerokeratitis: determining diagnostic criteria. Arch Ophthalmol. 1986; 104: 1313-1317.

54. Illingworth CD, Cook SD, Karabatsas CH, Easty DL. Acanthamoeba keratitis: risk factors and outcome. Br J Ophthalmol. 1995; 9(12): 1078-1082.

55. Khan NA, Tareen NK. Genotypic, phenotypic, biochemical, physiological and pathogenicity-based categorization of Acanthamoeba strains. Folia Parasitol. 2003; 50: 97-104.

56. Prasher P, Sachdeva P, Ravinder NB, Sachin W. Acanthamoeba keratitis: a review. NZOS 2004; 14(1): 1-7.

57. Galarza C, Ramos W, Gutierrez EL, Ronceros G, Teran M, Uribe M, et al. Cutaneous acanthamebiasis infection in immunocompetent and immunocompromised patients. Int J Dermatol. 2009; 48(12): 1324-1329

58. Walia R, Montoya JG, Visvesvera GS, Booton GC, Doyle RL. A case of successful treatment of cutaneous Acanthamoeba infection in a lung transplant recipient. Transpl Infect Dis. 2007; 9: 51-54.

59. Vargas-Zepeda J, Gómez-Alcalá AV, Vásquez-Morales JA, LiceaAmaya L, De Jonckheere JF, Lares-Villa F. Successful treatment of Naegleriafowleri meningoencephalitis by using intravenous amphotericin B, fluconazole and rifampicin. Arch Med Res. 2005; 36(1): 83-86.

60. Yagi S, Schuster FL, Visvesvara GS. Demonstration of Balamuthia and Acanthamoeba mitochondrial DNA in sectioned archival brain and other tissues by the polymerase chain reaction. Parasitol Res. 2008; 102(2): 211-217.

61. Selby DM, Chandra RS, Rakusan TA, Loechelt B, Markle BM, Visvesvara GS. Amebic Osteomyelitis in a Child with Acquired Immunodeficiency Syndrome: A Case Report. Fetal PediatrPathol. 1998; 18(1): 89-95.

62. Kaul DR, Lowe L, Visvesvara GS, Farmen S, Khaled YA, Yanik GA. Acanthamoeba infection in a patient with chronic graft-versus-host disease occurring during treatment with voriconazole. Transpl Infect Dis. 2008; 6: 437-441. 